This document is the accepted manuscript version of the following article:

Colvi11, E., Petersen, J. B. B., Hansen, R., Worm, E., Skouboe, S., Høyer, M., \& Pou1sen, P. R. (2018). Validation of fast motion-including dose reconstruction for proton scanning therapy in the liver. Physics in Medicine and Biology, 63(22), 225021 (9 pp.).

https://doi .org/10.1088/1361-6560/aaeae9

\title{
Validation of fast motion-including dose reconstruction for proton scanning therapy in the liver
}

\author{
Emma Colvill ${ }^{1,2}$, Jørgen BB Petersen ${ }^{3}$, Rune Hansen ${ }^{3}$, Esben Worm ${ }^{3}$, Simon Skouboe ${ }^{1}$, Morten Høyer ${ }^{4}$, Per R \\ Poulsen $^{1,5}$ \\ ${ }^{1}$ Department of Oncology, Aarhus University Hospital, Aarhus, Denmark \\ ${ }^{2}$ Center for Proton Therapy, Paul Scherrer Institute, Villigen, Switzerland \\ ${ }^{3}$ Department of Medical Physics, Aarhus University Hospital, Aarhus, Denmark \\ ${ }^{4}$ Danish Center for Particle Therapy, Aarhus University Hospital, Aarhus, Denmark \\ ${ }^{5}$ Department of Clinical Medicine, Aarhus University, Denmark
}

\section{Keywords}

dose reconstruction, liver, proton therapy, tumour motion

\section{Short title}

Fast motion-including proton PBS dose reconstruction

\section{Abstract}

This study validates a method of fast motion-including dose reconstruction for proton pencil beam scanning in the liver. The method utilizes a commercial treatment planning system (TPS) and calculates the delivered dose for any translational 3D target motion. Data from ten liver patients previously treated with photon radiotherapy with intrafraction tumour motion monitoring were used. The dose reconstruction method utilises an in-house developed program to incorporate beam's-eye-view tumour motion by shifting each spot in the opposite direction of the tumour and in-depth motion as beam energy changes for each spot. The doses are then calculated on a single CT phase in the TPS. Two aspects of the dose reconstruction were assessed: 1) The accuracy of reconstruction, by comparing dose reconstructions created using 4DCT motion with ground truth doses obtained by calculating phase specific doses in all 4DCT phases and summing up these partial doses. 2) The error caused by assuming 4DCT motion, by comparing reconstructions with 4DCT motion and actual tumour motion. The CTV homogeneity index $(\mathrm{HI})$ and the root-mean-square (rms) dose error for all dose points receiving $>70 \%,>80 \%$ and $>90 \%$ of the prescribed dose were calculated. The dose reconstruction resulted in mean (range) absolute CTV HI errors of 1.0\% (0.0 - 3.0)\% and rms dose errors of 2.5\% (1.0 - 5.3\%), 2.1\% (0.9$4.5 \%)$, and $1.8 \%(0.7-3.7 \%)$ for $>70 \%,>80 \%$ and $>90 \%$ doses, respectively, when compared with the ground truth. The assumption of 4DCT motion resulted in mean (range) absolute CTV HI errors of 5.9\% (0.0-15.0)\% and rms dose errors of $6.3 \%$ (3.9-12.6\%), 5.9\% (3.4-12.5\%), and $5.4 \%(2.6-12.1 \%)$ for $>70 \%,>80 \%$ and $>90 \%$ doses, respectively. The investigated method allows tumour dose reconstruction with the actual tumour motion and results in significantly smaller dose errors than those caused by assuming that motion at treatment is identical to the 4DCT motion. 


\section{Introduction}

Proton therapy allows improved high dose conformation when compared to photon radiotherapy, and pencil beam scanning (PBS) proton therapy can improve sparing of surrounding healthy tissues when compared to passive scattering proton therapy (Welsh et al., 2011; Zhang et al., 2018). For these reasons, there are indications for proton therapy for some liver tumours (Dionisi et al., 2014; Lee et al., 2014). However, intrafractional motion during proton PBS delivery can result in large dosimetric differences from the planned dose due to interplay effects (Bert et al., 2008; Zhang et al., 2012; Lambert et al., 2005). Methods have been developed to assess the interplay effects for liver proton deliveries including the use of 4DCT (Kraus et al., 2011; Zhang et al., 2012) or 4DMRI based motion models applied on CT scans (Bernatowicz et al., 2016). These methods use dose summation over different breathing phases by deformable image registration to obtain the tumour dose. However, deformable image registration is not easily validated and may lead to large errors in the reconstructed dose (Ribeiro et al., 2018).

Previous dose reconstruction methods can be time consuming and calculation intensive due to the use of multiple image sets and deformable image registration. Most often these methods use the 4DCT as the main basis for motion assessment assuming that the motion range and pattern will be the same throughout treatment. This assumption is often not accurate as amplitude and breathing patterns may change dramatically for some patients throughout treatment (Shah et al., 2013; von Siebenthal et al., 2007; Worm et al., 2018). A faster, simple method for liver target dose assessment that includes tumour motion would be manipulating the treatment plan to reflect target motion and using a commercially available treatment planning system (TPS) to provide a fast dose reconstruction in a single CT scan volume. A study which manipulated carbon ion PBS plans and calculated the motion-including dose on a single CT scan has been performed for prostate treatments (Ammazzalorso and Jelen, 2014).

In this study a similar spot-shift dose reconstruction method which uses a single 4DCT phase and the actual intra-treatment tumour motion is investigated for proton PBS for stereotactic body radiation therapy (SBRT) in the liver. The method has been developed from an isocentre shift method previously implemented for photon treatments (Poulsen et al., 2012) which utilizes a commercially available TPS. The aim of the study is to validate the method for liver proton PBS treatments by determining the dose reconstruction errors associated with the use of only a single 4DCT phase instead of all phases. Furthermore, the study determines the potential dose 
reconstruction errors by using the motion in the 4DCT scan instead of the actual tumour motion observed at treatment.

\section{Materials and methods}

\section{Spot-shift dose reconstruction method}

The spot-shift dose reconstruction method is similar to the method reported for carbon ion therapy of the prostate by Ammazzalorso and Jelen (2014). Unlike this previous implementation our implementation is proton specific and uses different software and computation systems. It utilizes an in-house developed computer program (Matlab 2015b) to incorporate target motion into proton PBS dicom plans exported from the TPS (Eclipse 13.6, Varian Medical Systems). Besides the treatment plan, the program also imports a target motion trajectory and a dicom structure set with the patient's body surface structure. The program simulates delivery of the treatment plan by assuming a layer switching time of $1.1 \mathrm{~s}$ and using approximate proton spot delivery durations and spot shift times of the Varian ProBeam facility at Maryland Proton Treatment Center (Poulsen et al., 2018). The simulation, combined with the motion trajectory, results in the target position during the delivery of each spot. If the delivery duration of a spot exceeds a user-defined time resolution $(50 \mathrm{~ms}$ in this study) the spot is divided into sub-spots that may be given different target positions to allow modelling of target motion during the spot delivery.

The computer program incorporates the target motion into the dicom treatment plans by shifting each proton spot to its position as seen in tumour's eye view, while depth motion is modelled by modifying the beam energy. As an example, a target shift to the left and to a more shallow depth at the time of delivery of a spot is modelled by moving the spot to the right and increasing its energy as illustrated in Figure 1 . The energy is also modified for depth changes caused by a different source-surface-distance at the new entrance point of the spot than at the original entrance position (Figure 1). Here, the dicom body surface structure is used to calculate the source-surface-distance at each spot position, which means that the body surface is assumed to be static while the target follows the specified motion trajectory inside the body structure. The pencil beam energy modifications to emulate target depth motion approximates the proton range as follows(Gottschalk, 2012):

$R=0.000244 E^{1.75}(1)$ 
Where $\mathrm{R}$ is the range in $\mathrm{g} / \mathrm{cm}^{2}$ and $\mathrm{E}$ is the pencil beam energy in MeV. The energy modification assumes water density inside the body contour when calculating the proton range changes. All energy changes are accompanied by monitor unit (MU) adjustments to maintain the original number of protons in the pencil beam. The spot-shift dose reconstruction can readily include interplay effects for a field by applying different shifts to each individual spot, or even to sub-spots, depending on the target position at the time of delivery of the spot.

The manipulated dicom plans are then imported back into the TPS for motion-including dose calculation, decoupling the motion incorporation and the dose calculation. The motion incorporation by the in-house computer program took 1-3 minutes per plan on a standard desktop CPU.

\section{Treatment plans and simulations}

This study consists of two parts as shown in Figure 2 and described in the following sections. In both parts, the spot-shift dose reconstruction was investigated for ten liver patients previously treated with photon SBRT with internal tumour motion monitoring during treatment delivery by implanted electromagnetic transponders (Calypso, Varian Medical Systems, Palo Alto, CA)(Poulsen et al., 2015; Worm et al., 2018). This study included twelve patients who had been included in the Calypso protocol at the time of the study except for two patients with large artefacts in the 4DCT scan. For each patient, the location of the three Calypso transponders and their centroid was determined on all ten phases of a 4DCT scan. Since the transponders may not be used for proton treatments due to their high density, they were segmented and their Hounsfield Units (HU) overwritten for the purpose of proton dose calculation. The HU assigned for each patient varied between 40 and 95 and was selected as an average of the surrounding liver HU. The clinical target volumes (CTV) were defined on a breathhold contrast enhanced CT scan acquired before the 4DCT scan and the shift of the CTV on each CT phase was assumed to be the same as the translational shift of the Calypso transponder centroid relative to the exhale phase. Of the ten included patients, nine had a unifocal tumour while one patient had three metastases. For the latter patient, only the single largest contoured CTV (which was also the metastasis closest to the transponders) was used for this study. An internal CTV (ICTV) was created from the union of the CTVs of all phases. For the purpose of this study, a planning target volume (PTV) approach was used and a PTV for optimization was created by expanding the ICTV with $5 \mathrm{~mm}$ margins in each direction. An SBRT proton treatment plan with a prescription dose of 56.25Gy (RBE) in 3 fractions was created on the exhale phase for each patient. Each plan used two fields, single-field-optimization (SFO) with $6 \mathrm{~mm}$ spot spacing and normalization of $100 \%$ of the prescription dose to $95 \%$ of the ICTV volume. 


\section{Ground truth Dose reconstruction (shifted target) method (shifted dose)}

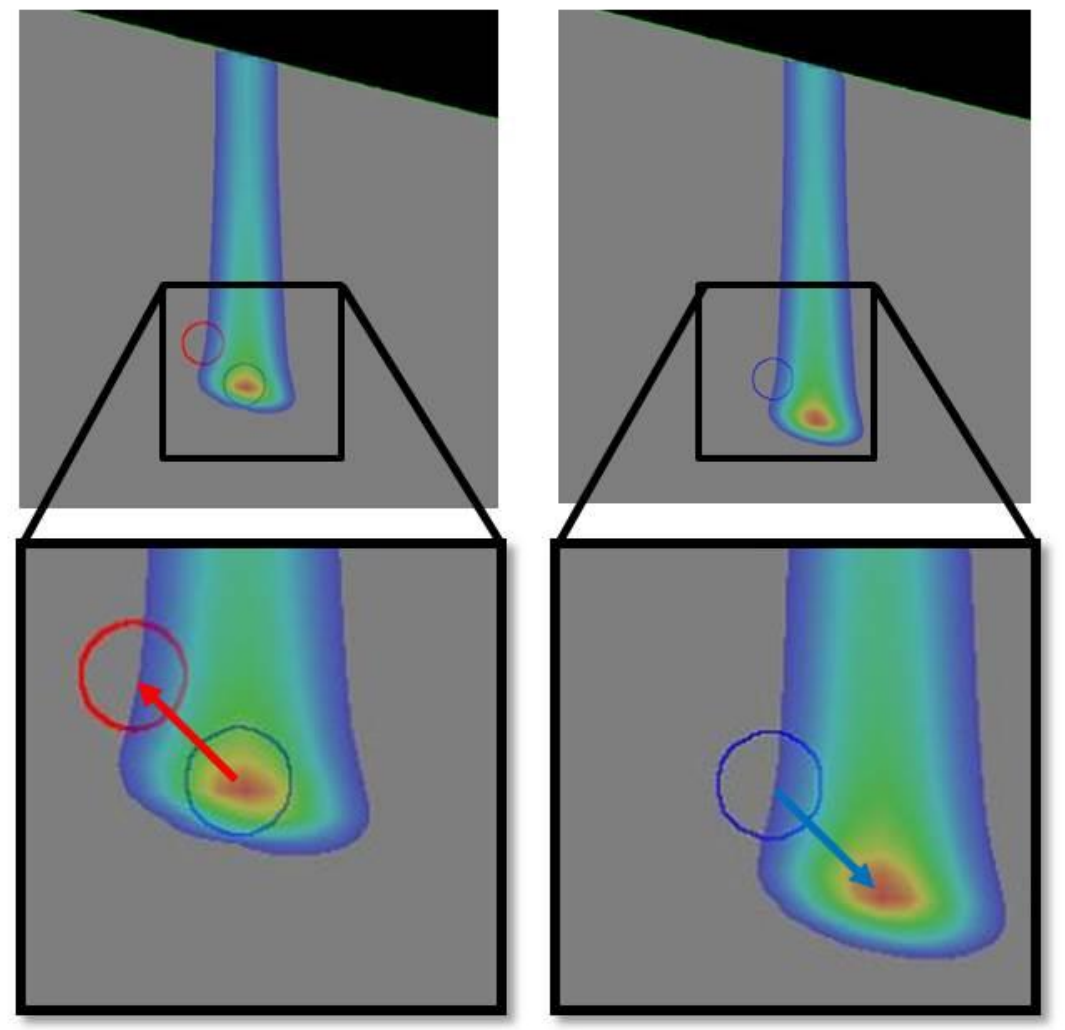

Figure 1. The basic mechanism of the spot-shift method for proton pencil beam scanning dose reconstruction illustrated for a single pencil beam in a digital water phantom. Left: A $10 \mathrm{~mm}$ diameter spherical target (blue structure) moves along the red arrow to the red structure, i.e. $10 \mathrm{~mm}$ upwards and $10 \mathrm{~mm}$ towards left. Right: The dosimetric effect of the target motion is emulated by shifting the spot in the opposite direction along the blue arrow, i.e. by increasing the pencil beam energy to increase the range by $10 \mathrm{~mm}$ and shifting the position $10 \mathrm{~mm}$ to the right. An additional energy correction is made to compensate for the sloped surface at the beam entrance.

\section{Part 1: Accuracy of spot-shift dose reconstruction method for liver SBRT}

The spot-shift dose reconstruction neglects that the pencil beams enter through different anatomies with different water equivalent path lengths as the tumour moves inside the patient. Furthermore, the emulation of in-depth motion by energy modifications assumes water density and neglects changes in the Bragg peak shape and pencil beam width with energy. The consequence of these effects was investigated by comparing the spot- 
shift dose reconstruction performed in the exhale phase with a ground truth dose reconstruction calculated by use of all ten 4DCT phases (Figure 2, left). In both dose reconstructions, the liver tumour was assumed to move in a cyclic manner as the Calypso transponder centroid in the 4DCT scan with a period length of $4 \mathrm{~s}$ and $0.4 \mathrm{~s}$ pause in each phase.

Spot-shift dose reconstruction plans were created by shifting the position and energy of each individual spot in the opposite direction to the target position at the time of spot delivery. All spot shifts were made relative to the exhale phase and the motion-including dose was reconstructed by importing the spot-shift plan into the TPS and calculating it on the exhale phase.

The ground truth dose reconstruction was made by splitting the original treatment plan (dicom exported from Eclipse) into 10 partial plans with the spots, which would have been delivered in the corresponding 4DCT phases. The partial plans were imported into the TPS and each calculated on the corresponding 4DCT phase. The dose distributions from the partial plans were then exported, shifted rigidly according to the transponder centroid shift relative to the exhale phase, summed and imported into the exhale 4DCT phase for comparison with the spot-shift dose reconstruction plan. The ground truth dose thus accounted for anatomical changes with respiration, but assumed that the CTV moved rigidly in the liver with the transponder centroid in order to avoid uncertainties from deformable image registration.

The dosimetric effect of motion was compared between the spot-shift dose reconstructions and the ground truth by calculating the CTV homogeneity index $\left(H I(\%)=\frac{D_{2 \%}-D_{98 \%}}{D_{\text {mean }}} * 100\right)$ of the original plan, the spot-shift dose reconstruction, and the ground truth dose reconstruction. Here, $\mathrm{D}_{\mathrm{x} \%}$ denotes the dose delivered to $\mathrm{x} \%$ of the CTV while $D_{\text {mean }}$ is mean dose to the CTV. Furthermore, the rms dose difference between the spot-shift dose reconstruction and the ground truth dose reconstruction was calculated for dose points above $70 \%, 80 \%$ and $90 \%$ cut-off levels of the prescription dose. 


\section{Part 1: Accuracy of spot-shift Part 2: Accuracy of 4DCT motion dose reconstruction \\ assumption}
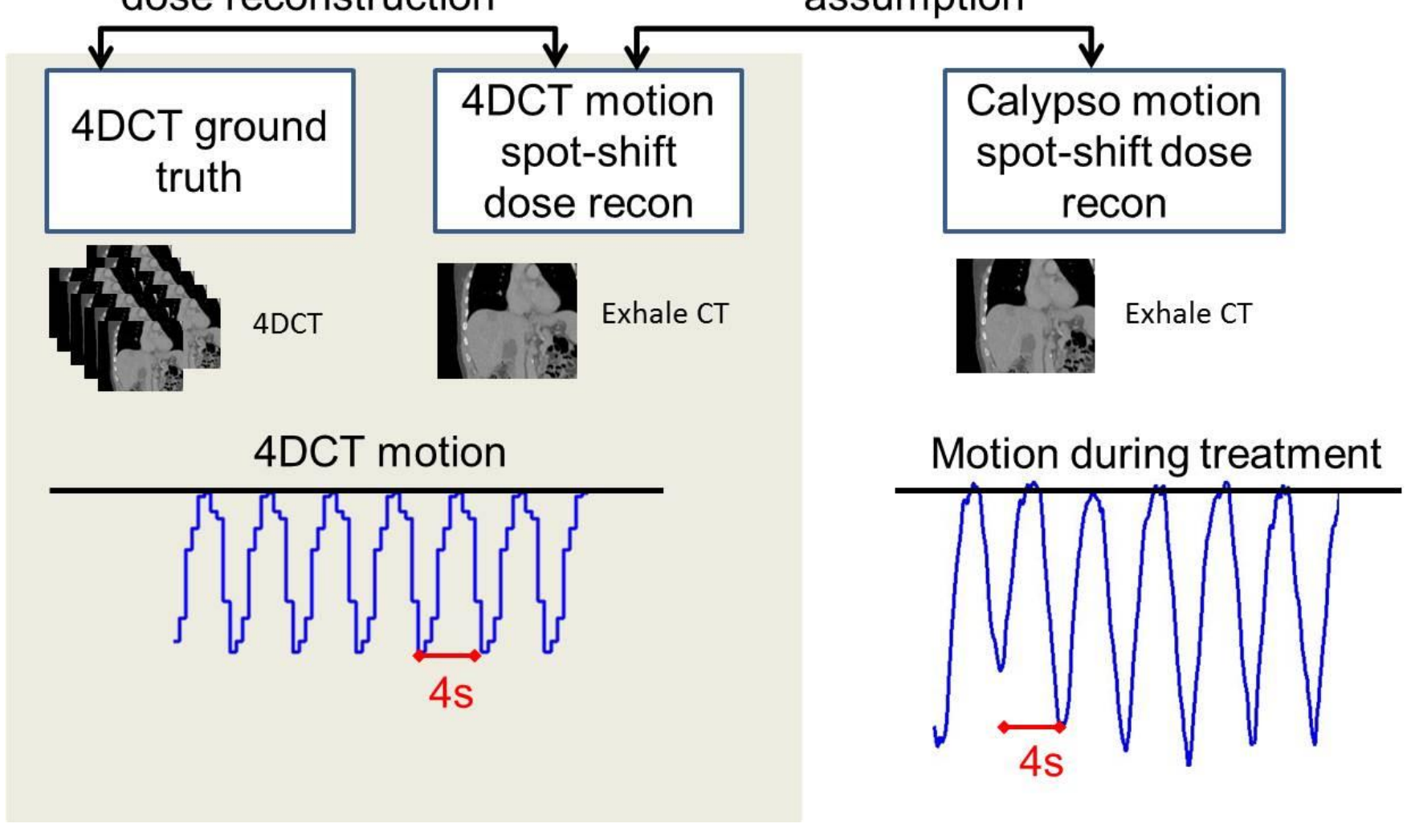

Figure 2. Study overview. Part 1 investigates the accuracy of the spot-shift dose reconstruction method for liver SBRT. Part 2 investigates the accuracy of using the 4DCT motion instead of the actual motion at treatment in the motion-including dose reconstruction.

\section{Part 2: Spot-shift dose reconstruction with actual tumour motion}

The dose summation method used to generate the ground truth is able to account for irregular breathing periods by sorting of the spots differently into the ten 4DCT phases, but the method cannot account for other motion amplitudes than the one recorded in the 4DCT scan. The spot-shift method does not have this limitation as it can account for arbitrary tumour motion. The dosimetric error by assuming the same motion amplitude and breathing trajectory shape during treatment as in the 4DCT scan was investigated with spotshift dose reconstructions in Part 2 of the study by comparing dose reconstructions made with the 4DCT motion and with the actual tumour motion measured with Calypso during treatment delivery (Figure 2, right). The Calypso motion dose reconstruction used the motion trajectory observed for a single fraction of each 
patient(Worm et al., 2018). The motion for the first proton PBS field was taken from the time point immediately after cone-beam CT guided patient setup, while the motion during the second field was taken from later in the same fraction. The timescale of the Calypso-recorded trajectory for each individual breathing cycle was adjusted such that all full inhales in the cranio-caudal direction occurred with 4 s intervals in order to ensure that each spot was delivered in the same phase with the Calypso motion as with the 4DCT motion (Figure 2, right). Furthermore, the mean exhale level of the Calypso-recorded trajectory was set to zero in all three directions to match the exhale level of the 4DCT motion. In this way, the two dose reconstructions, i.e. with 4DCT motion and with Calypso motion, would result in identical dose distributions if the tumour motion at treatment were identical to the 4DCT motion except for breathing period variations. Any difference between the two reconstructed doses thus reflects a different tumour motion trajectory shape at treatment compared to the 4DCT. The spot-shift dose reconstruction began the spot delivery simulation at the exhale phase, matching that of the ground truth and 4DCT motion spot-shift dose reconstructions.

The mean and standard deviation of the difference between the Calypso-recorded breathing motion ranges and the 4DCT motion range were calculated for each motion axis. The spot-shift dose reconstructions with 4DCT motion and with Calypso-recorded motion were compared by calculating the CTV HI of both dose reconstructions as well as the rms dose difference between the two dose reconstructions for all dose calculations points receiving $>70 \%,>80 \%$ and $>90 \%$ of the prescribed dose. Statistically significant differences between the rms dose errors of the spot-shift method (Part 1) and the assumption of 4DCT motion at treatment (Part 2) were tested using a two-tailed Wilcoxon rank sum test.

\section{Results}

\section{Part 1: Accuracy of spot-shift dose reconstruction method for liver SBRT}

The ten patients had varying tumour sizes, locations and 4DCT motion ranges as summarized in Table 1 . The mean (range) of the ICTV volume was $78.5(2.5-249.8) \mathrm{cm}^{3}$. Three patients had tumours in the superior part of the liver bordering on the diaphragm, three in an anterior location, three centrally and one laterally. 


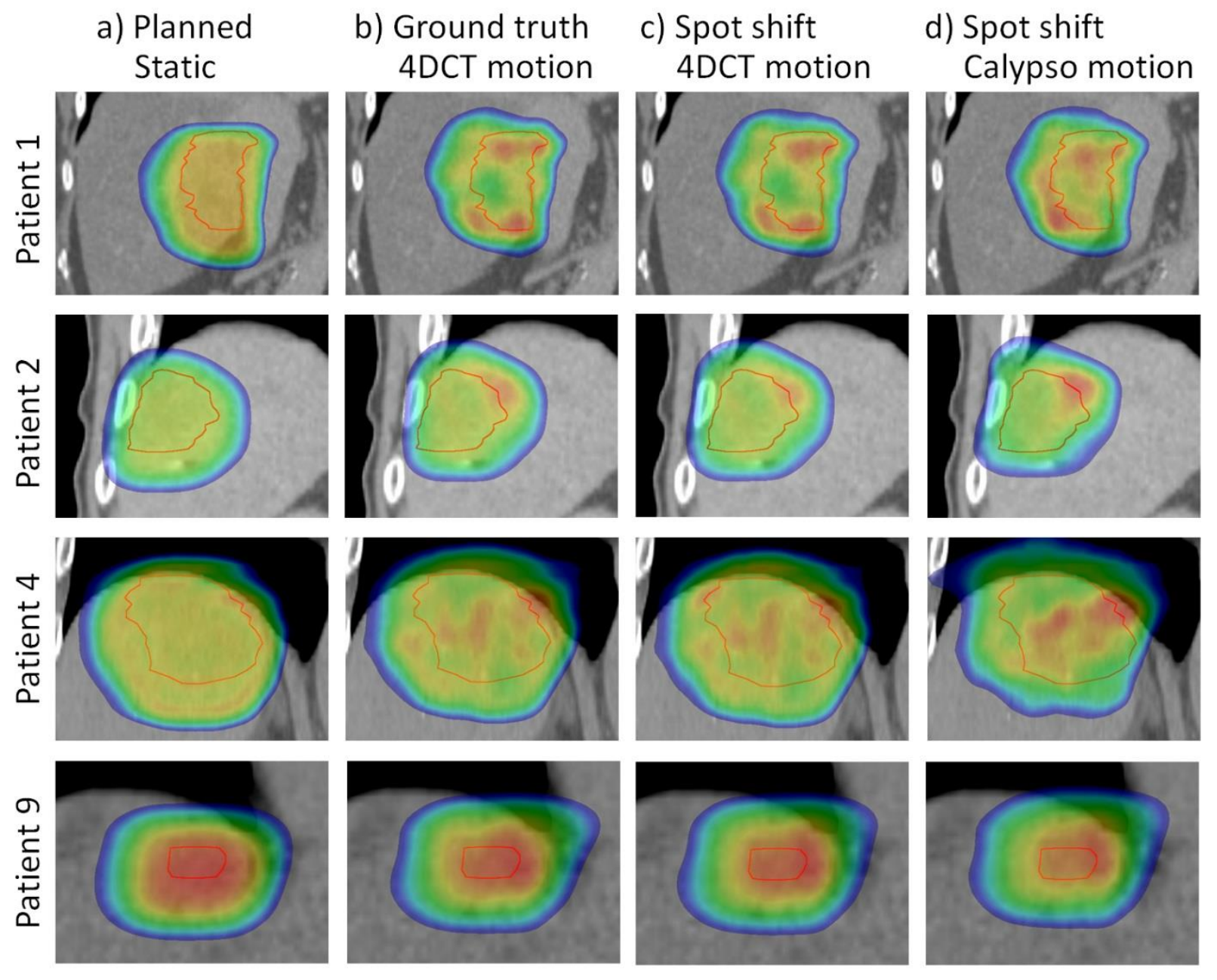

Figure 3. Examples of dose distributions in the coronal plane through the centre of the CTV (red structure) for four patients for a) the original treatment plan, $b$ ) the ground truth dose reconstruction with 4DCT motion, c) the spot-shift dose reconstruction with 4DCT motion and d) the spot-shift dose reconstruction with the motion at treatment. The dose colour wash range is 50-117\% (Patients 1 and 4), 50-125\% (Patient 2) and 50-103\% (Patient 9). 
(a) Patient 1

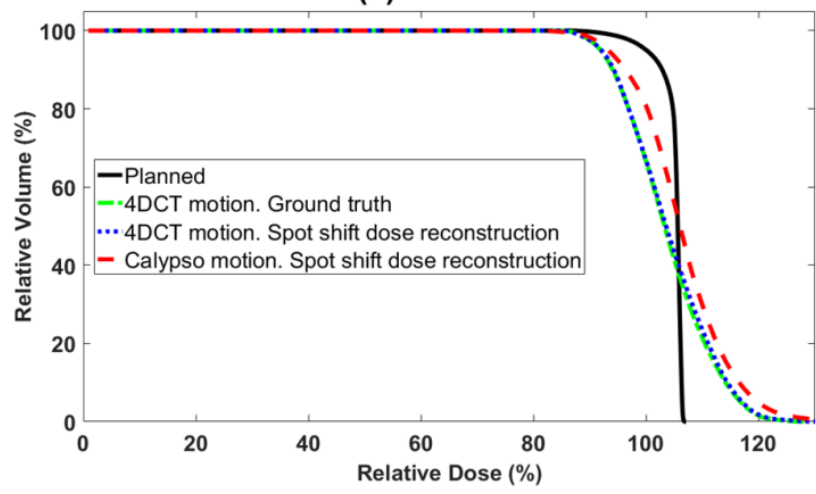

(c) Patient 4

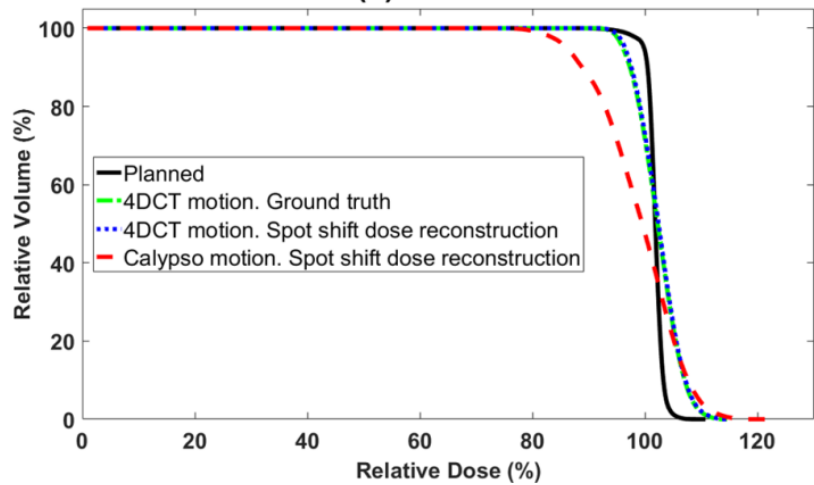

(b) Patient 2

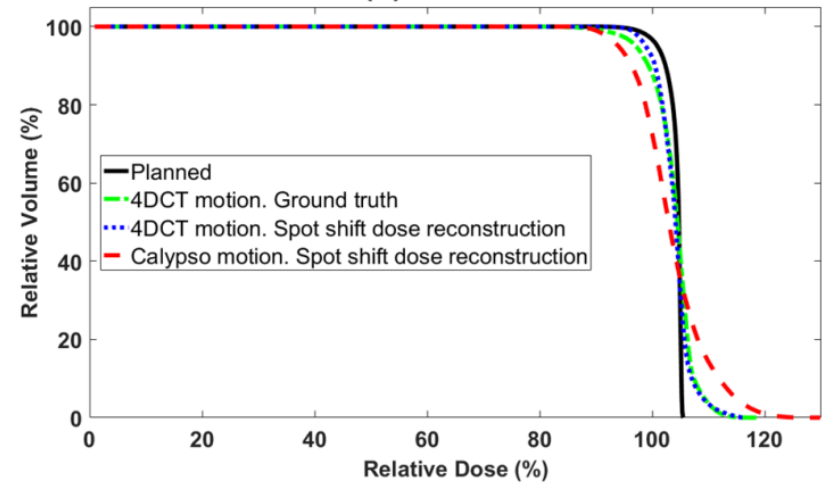

(d) Patient 9

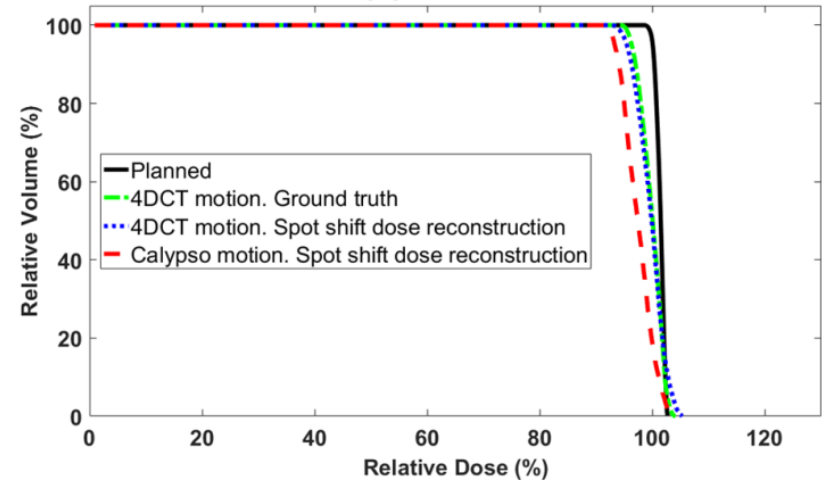

Figure 4. Dose volume histograms for the clinical target volume for the dose calculations presented in Figure 3 with the original static treatment plan (black), the ground truth with 4DCT motion (green), and the spot-shift dose reconstruction with 4DCT motion (blue) and motion at treatment (red).

The motion-including dose reconstructions are illustrated for four patients in Figure 3 while Figure 4 shows the corresponding CTV dose volume histograms. There was in general good agreement in the patterns of interplay effects between the spot-shift dose reconstruction with 4DCT motion and the ground truth as seen by Figures $3 \mathrm{~b}$ and $3 \mathrm{c}$. The mean (and range) of the rms error of the spot-shift dose reconstruction with 4DCT motion for all ten patients was $2.5 \%(1.0-5.3 \%)$ for dose points greater than $70 \%, 2.1 \%(1.0-4.5 \%)$ for dose points greater than $80 \%$, and $1.8 \%(0.7-3.7 \%)$ for dose points greater than $90 \%$ (Table 1$)$. The rms error was largest for Patients 1 and 2 who are both presented in Figures 3 and 4 . The CTV HI for the original static plan and the motion-including dose reconstructions are presented for all patients in Figure 5. The mean (and range) absolute difference in the $\mathrm{HI}$ between the spot-shift dose reconstruction with 4DCT motion and the ground truth was $1.0 \%(0.0-3.0) \%$. The difference was largest for Patients 2 and 9, who are both presented in Figures 3 and 4. 
Table 1. Statistics for all 10 patients including the tumour volume and location, 4DCT motion range in the superior-inferior (SI), anterior-posterior (AP) and left-right (LR) directions as well as dose errors of the spot-shift dose reconstruction with the 4DCT motion including the rms errors relative to the ground truth for dose points $>70 \%,>80 \%$ and $>90 \%$ of the prescription dose.

\begin{tabular}{|c|c|c|c|c|c|c|c|c|}
\hline \multirow[t]{2}{*}{$\begin{array}{l}\text { Patient } \\
\text { number }\end{array}$} & \multirow[t]{2}{*}{$\begin{array}{l}\text { ICTV } \\
\text { volume } \\
\left(\mathrm{cm}^{3}\right)\end{array}$} & \multirow[t]{2}{*}{$\begin{array}{l}\text { Tumour } \\
\text { location in } \\
\text { liver }\end{array}$} & \multicolumn{3}{|c|}{ 4DCT motion range $(\mathrm{mm})$} & \multicolumn{3}{|c|}{$\begin{array}{l}\text { rms error of spot-shift dose } \\
\text { reconstruction (relative to } \\
\text { ground truth) (\%) }\end{array}$} \\
\hline & & & SI & AP & LR & $>70 \%$ & $>80 \%$ & $>90 \%$ \\
\hline 1 & 97.8 & central & 16.0 & 6.7 & 1.9 & 4.85 & 4.25 & 3.60 \\
\hline 2 & 37.1 & lateral & 8.8 & 0.8 & 2.1 & 5.25 & 4.54 & 3.69 \\
\hline 3 & 34.4 & central & 6.9 & 1.6 & 0.4 & 0.98 & 0.95 & 0.90 \\
\hline 4 & 203.2 & superior & 12.0 & 4.8 & 3.4 & 2.34 & 2.14 & 1.78 \\
\hline 5 & 249.8 & anterior & 13.6 & 7.5 & 3.5 & 2.58 & 2.19 & 1.83 \\
\hline 6 & 32.2 & ant-central & 8.7 & 2.6 & 1.7 & 2.01 & 1.94 & 1.90 \\
\hline 7 & 2.8 & anterior & 6.5 & 1.3 & 1.3 & 1.32 & 1.08 & 0.95 \\
\hline 8 & 89.1 & superior & 8.3 & 3.4 & 1.1 & 2.38 & 2.17 & 1.91 \\
\hline 9 & 2.5 & superior & 5.9 & 4.9 & 0.3 & 2.38 & 1.26 & 1.18 \\
\hline 10 & 36.0 & central & 9.4 & 2.3 & 1.0 & 1.18 & 0.95 & 0.70 \\
\hline Mean & 78.5 & - & 9.7 & 3.6 & 1.7 & 2.5 & 2.1 & 1.8 \\
\hline
\end{tabular}

\section{Part 2: Spot-shift dose reconstruction with actual tumour motion}

The superior-inferior tumour motion range measured with Calypso at treatment was in general larger than the 4DCT motion range (Table 2). The mean SI motion range at treatment agreed with the 4DCT motion range within $1 \mathrm{~mm}$ for three patients and exceeded the 4DCT motion range with at least $3 \mathrm{~mm}$ for five patients. There were also varying standard deviations in the trajectory ranges reflecting the irregularity of the breathing. The 
largest discrepancy between tumour motion range at treatment and at 4DCT scanning occurred for Patients 2 and 4, who are both included in Figures 3 and 4. The actual motion at treatment resulted in markedly different interplay effects than predicted from the 4DCT motion as seen by comparing Figures $3 \mathrm{c}$ and $3 \mathrm{~d}$. For patient 4 , the CTV coverage was also highly affected by the actual motion during treatment (Figure 4c). The rms error by assuming 4DCT motion at treatment was significantly larger than the spot-shift dose reconstruction errors of Part 1 with mean (ranges) of 6.3\% (3.9-12.6\%)(p-value $=0.005)$ for dose points greater than 70\%, 5.9\% (3.4$12.5 \%)$ ( $p$-value $<0.005)$ for dose points greater than $80 \%$, and $5.4 \%(2.9-12.1 \%)$ ( $p$-value $<0.005)$ for dose points greater than 90\% (Table 2). The CTV HI for the spot-shift dose reconstruction with the Calypso-measured motion is compared with the other dose reconstructions for each patient in Figure 5. The mean (and range) absolute difference in the $\mathrm{HI}$ between the spot-shift dose reconstruction with Calypso motion and with 4DCT motion was $5.9 \%(0-15) \%$.

Table 2. Statistics for all 10 patients in the study including the 4DCT superior-inferior (SI) motion range and the Calypso trajectory range difference from $4 D C T$ in the SI, anterior-posterior (AP) and left-right (LR) directions as well as dose errors caused by assuming 4DCT motion at treatment including the rms errors relative to the calypso spot shift dose reconstruction for dose points $>70 \%,>80 \%$ and $>90 \%$ of the prescription dose.

\begin{tabular}{|c|c|c|c|c|c|c|c|}
\hline \multirow[t]{2}{*}{$\begin{array}{l}\text { Patient } \\
\text { number }\end{array}$} & \multirow{2}{*}{$\begin{array}{c}\text { 4DCT } \\
\text { motion SI } \\
(\mathrm{mm})\end{array}$} & \multicolumn{3}{|c|}{$\begin{array}{l}\text { Mean (standard deviation) motion } \\
\text { range difference between treatment } \\
\text { and } 4 \mathrm{DCT}(\mathrm{mm})\end{array}$} & \multicolumn{3}{|c|}{$\begin{array}{l}\text { rms error of 4DCT motion } \\
\text { assumption (relative to Calypso spot- } \\
\text { shift reconstruction) (\%) }\end{array}$} \\
\hline & & $\mathrm{SI}$ & $A P$ & LR & $>70 \%$ & $>80 \%$ & $>90 \%$ \\
\hline 1 & 16 & $0.5( \pm 3.1)$ & $0.3( \pm 1.3)$ & $1.9( \pm 1.0)$ & 6.27 & 6.26 & 6.15 \\
\hline 2 & 8.8 & $4.6( \pm 3.8)$ & $4.2( \pm 1.7)$ & $0.3( \pm 0.5)$ & 9.6 & 9.21 & 8.38 \\
\hline 3 & 6.9 & $0.7( \pm 3.7)$ & $1.7( \pm 2.3)$ & $-0.1( \pm 0.4)$ & 4.39 & 4.01 & 3.59 \\
\hline 4 & 12 & $5.4( \pm 5.3)$ & $4.1( \pm 2.7)$ & $4.5( \pm 0.5)$ & 12.55 & 12.47 & 12.07 \\
\hline 5 & 13.6 & $1.4( \pm 2.1)$ & $1.4( \pm 1.3)$ & $1.4( \pm 0.8)$ & 8.81 & 7.94 & 6.83 \\
\hline 6 & 8.7 & $4.1( \pm 0.9)$ & $1.0( \pm 0.3)$ & $1.0( \pm 0.4)$ & 4.78 & 4.51 & 4.11 \\
\hline 7 & 6.5 & $3.8( \pm 1.4)$ & $2.2( \pm 0.5)$ & $1.4( \pm 0.4)$ & 4.19 & 3.54 & 2.64 \\
\hline 8 & 8.3 & $3.5( \pm 1.6)$ & $2.6( \pm 1.2)$ & $1.8( \pm 0.9)$ & 4.8 & 4.62 & 4.36 \\
\hline 9 & 5.9 & $2.1( \pm 1.1)$ & $-0.1( \pm 0.6)$ & $1.0( \pm 0.3)$ & 3.85 & 3.4 & 3.04 \\
\hline 10 & 9.4 & $-0.7( \pm 4.6)$ & $-0.7( \pm 1.5)$ & $-0.4( \pm 0.9)$ & 3.95 & 3.42 & 2.9 \\
\hline
\end{tabular}




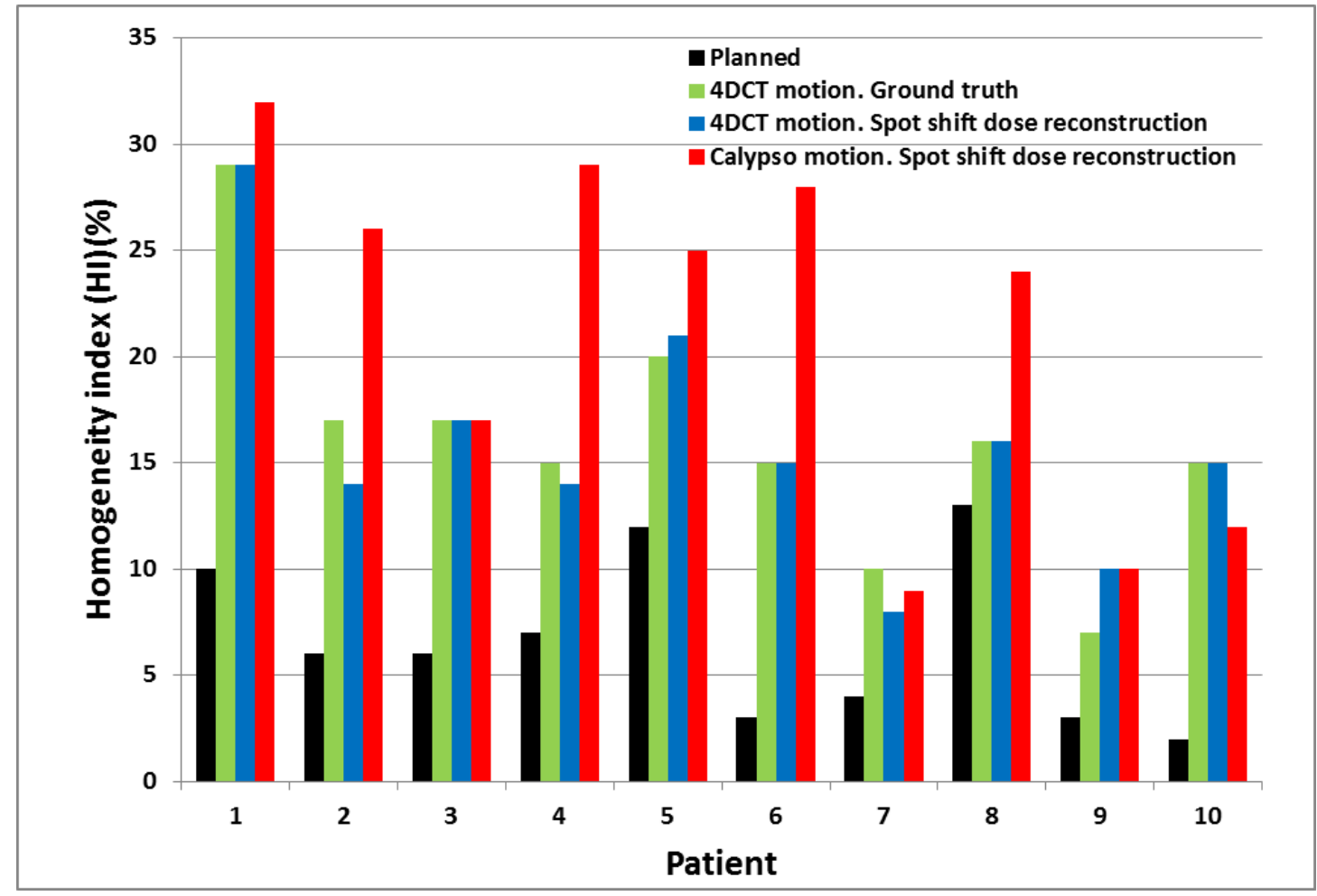

Figure 5. The homogeneity index (HI) of the CTV for the original static treatment plan (black), the ground truth with 4DCT motion (green), and the spot-shift dose reconstruction with 4DCT motion (blue) and motion at treatment (red).

\section{Discussion}

This study investigated a simple spot-shift method for motion-including dose reconstruction of proton PBS treatments in the liver. The dose reconstruction works for any translational tumour motion and is fast and easy to automate since it is performed in a single CT phase without deformable image registration. The issue of interplay effects is pronounced for proton and ion beam scanning due to the nature in which the dose is deposited in tissue(Bert et al., 2008; Dowdell et al., 2013a). The comparison with ground truth doses showed that the error in the spot-shift dose reconstruction method is small for liver plans even for patients with large motion ranges. Our results are in agreement with a previous water phantom and prostate study 
(Ammazzalorso and Jelen, 2014) which used a similar spot-shift dose reconstruction method for carbon ions and showed similarly small errors as the current study although with smaller motion and with minimal changes in the radiological path lengths associated with pelvic targets.

In the high dose region above $80 \%$, which forms the basis for interplay effect calculations in the target region, the rms dose error of the spot-shift method (Table 1) was in mean 36\% (range 17-68\%) of the error caused by assuming 4DCT motion at treatment delivery (Table 2). The variations between patients in the spot-shift dose reconstruction accuracy are likely due to a number of factors including tumour location and size as well as 4DCT motion range. No significant correlation was found between tumour motion range or tumour size and the rms dose error. As seen in Table 1, the largest rms errors occurred for Patient 1, who had the largest motion range and Patient 2, who had much smaller motion but a tumour location close to the ribs (Figure 3 ). The spotshift dose reconstruction is expectedly less accurate in regions with large density variations.

The differences between the spot-shift dose reconstruction with the 4DCT motion and the Calypso-measured motion at treatment (Part B) are caused by differences in both the mean motion range and its variability (standard deviation) (Table 2). Using doses above $80 \%$ as an example it was found that neither the mean motion range difference $(p=0.14, r=0.51)$ nor the motion range variability $(p=0.09, r=0.59)$ correlated significantly with the rms dose difference between 4DCT motion and Calypso-measured motion. On the other hand, the sum of the mean motion range difference and the variability did correlate significantly with the rms dose difference $(p=0.01, r=0.76)$. It indicates that the actually delivered dose and thus the interplay effects depends on the detailed motion at treatment and therefore cannot be predicted reliably by dose reconstruction methods that only encompass the 4DCT motion.

The challenges with interplay effects are most pronounced in treatment sites such as liver and lung due to differences in electron density between tissues and the amplitude and complexity of the target motion(Dowdell et al., 2013b; Kraus et al., 2011). Most studies involving dose calculation for lung and liver proton therapy use DIR techniques(Bernatowicz et al., 2016; Ribeiro et al., 2018) which can be time and resource consuming and include uncertainties of the DIR themselves. A previous study did compare DIR to rigid motion dose reconstruction for liver patients(Knopf et al., 2012) and found some discrepancies between the two, mostly on the distal edge of the deliveries. In the current study we did not use DIR for the creation of the ground truth as we chose to avoid DIR-related uncertainties in our comparison between the spot-shift dose reconstruction and the ground truth. There are limitations, however, in not using DIR; the calculated motion- 
including ground truth dose reliably represents the dose delivered to points that move rigidly with the transponder centroid. However, if the liver tumour motion is more complex and includes deformations then the tumour will receive a different dose as a function of time.

The dose of the spot-shift method is only correct for anatomy that moves rigidly with the tumour. The spotshift dose reconstruction is therefore very suitable to assess interplay effects in the tumour, while risk organ doses should be interpreted more carefully, and reconstructed doses to e.g. ribs and skin that move very differently from the tumour will be error prone. Besides non-modelled path length changes, there are also errors caused by differences in the Bragg peak shape with entrance energy which is also present even in a homogeneous medium such as in Figure 1, where the Bragg peak shape of the shifted spot differs from the original spot.

In this current implementation, deformation of the target itself is not taken into account. How much this affects the accuracy of the target dose will need further investigation, however, identification of target motion within the liver using $\mathrm{CT}$ is often difficult. The heterogeneity of dose using the spot shift dose reconstruction method is seen not only at the edges of the target but also in the centre. This, along with the density of surrounding tissues being similar to the tumour means that the effects of deformation of the target are not expected to be highly clinically relevant. Deformation of the target itself is likely to be more clinically relevant for potential future implementations such as lung where the density difference between the target and surrounding tissue is much greater. Deformations can be included in the spot-shift dose reconstruction when the deformations occur on a length scale larger than the individual spots, namely by applying additional shifts to each spot, thereby modelling how the tissue at the Bragg peak has moved due to both rigid and non-rigid tumour motion. However, the spot-shift method cannot handle deformations on a smaller length scale that result in deformation of the dose received in the tissue from single spots. The method could be expanded to encompass multiple targets with differential motion by applying different shifts to spots with Bragg peaks in the different targets. The method could also be improved by including the actual tissue density rather than water density when depth motion is emulated as beam energy changes. This will, however, complicate the method and cause deviations from the intended simplicity that allows easy automation, e.g. for large scale simulations for statistical analysis of interplay effects. For this particular study, the authors decided to focus on the ease and speed of the process compared to DIR 4DCT based methods in achieving accurate target dose reconstructions. The relatively large dose reconstruction error for Patient 2, who had a tumour close to the ribs 
(Figure 2, Table 1) shows that the spot-shift dose reconstruction is less accurate for tumours surrounded by tissue with density variations, which may indicate less accuracy in the lung than in the liver.

This relatively fast and simple spot-shift dose reconstruction method could be useful in the evaluation of different delivery and motion management methods for proton PBS prior to and during commissioning of new techniques as well as a patient-specific quality assurance tool of the actual dose delivered during proton therapy.

\section{Conclusion}

A fast spot-shift method of dose reconstruction has been developed and this study shows that for liver the errors associated with non-modelled anatomy changes are small. The ability to include the actual tumour motion allows a significant gain in dose reconstruction accuracy when the motion range at treatment differs from the 4DCT motion.

\section{Acknowledgements}

This study was supported by grants from the Danish Cancer Society and Varian Medical Systems. Dr Colvill has received funding from the European Union's Horizon 2020 research and innovation programme under the Marie Skłodowska-Curie grant agreement No 701647. The authors gratefully thank Professor Katja Langen and colleagues at Maryland Proton Treatment Center for sharing their proton beam data for the treatment planning in this study.

\section{References}

Ammazzalorso F and Jelen U 2014 A 4D dose computation method to investigate motion interplay effects in scanned ion beam prostate therapy Physics in medicine and biology 59 N91

Bernatowicz K, Peroni M, Perrin R, Weber D C and Lomax A 2016 Four-dimensional dose reconstruction for scanned proton therapy using liver 4DCT-MRI International Journal of Radiation Oncology* Biology* Physics 95 216-23

Bert C, Grözinger S O and Rietzel E 2008 Quantification of interplay effects of scanned particle beams and moving targets Physics in medicine and biology 532253

Dionisi F, Widesott L, Lorentini S and Amichetti M 2014 Is there a role for proton therapy in the treatment of hepatocellular carcinoma? A systematic review Radiotherapy and Oncology 111 1-10

Dowdell S, Grassberger C and Paganetti H 2013a Four-dimensional Monte Carlo simulations demonstrating how the extent of intensity-modulation impacts motion effects in proton therapy lung treatments Medical physics $\mathbf{4 0}$ 
Dowdell S, Grassberger C, Sharp G and Paganetti H 2013b Interplay effects in proton scanning for lung: a 4D Monte Carlo study assessing the impact of tumor and beam delivery parameters Physics in medicine and biology 584137

Gottschalk B 2012 Physics of proton interactions in matter. In: Proton Therapy Physics, ed H Paganetti (Boca Raton: CRC Press, Taylor \& Francis Group) pp 19-59

Knopf A, Zhan Y, Boye D and Lomax A 2012 How realistic is 4D treatment planning for scanned proton therapy using rigid motion models? Strahlentherapie Und Onkologie 188 732-

Kraus K, Heath E and Oelfke U 2011 Dosimetric consequences of tumour motion due to respiration for a scanned proton beam Physics in medicine and biology 566563

Lambert J, Suchowerska N, McKenzie D and Jackson M 2005 Intrafractional motion during proton beam scanning Physics in medicine and biology 504853

Lee S U, Park J-W, Kim T H, Kim Y-J, Woo S M, Koh Y-H, Lee W J, Park S-J, Kim D Y and Kim C-M 2014 Effectiveness and safety of proton beam therapy for advanced hepatocellular carcinoma with portal vein tumor thrombosis Strahlentherapie und Onkologie 190 806-14

Poulsen P R, Eley J, Langner U, Simone II C B and Langen K 2018 Efficient Interplay Effect Mitigation for Proton Pencil Beam Scanning by Spot-Adapted Layered Repainting Evenly Spread out Over the Full Breathing Cycle International Journal of Radiation Oncology • Biology • Physics 100 226-34

Poulsen P R, Schmidt M L, Keall P, Worm E S, Fledelius W and Hoffmann L 2012 A method of dose reconstruction for moving targets compatible with dynamic treatments Medical Physics 39 6237-46

Poulsen P R, Worm E S, Hansen R, Larsen L P, Grau C and Høyer M 2015 Respiratory gating based on internal electromagnetic motion monitoring during stereotactic liver radiation therapy: First results Acta Oncologica 54 1445-52

Ribeiro C O, Knopf A, Langendijk J A, Weber D C, Lomax A J and Zhang Y 2018 Assessment of dosimetric errors induced by deformable image registration methods in $4 \mathrm{D}$ pencil beam scanned proton treatment planning for liver tumours Radiotherapy and Oncology

Shah A P, Kupelian P A, Waghorn B J, Willoughby T R, Rineer J M, Mañon R R, Vollenweider M A and Meeks S L 2013 Real-Time Tumor Tracking in the Lung Using an Electromagnetic Tracking System International Journal of Radiation Oncology*Biology*Physics 86 477-83

von Siebenthal M, Szekely G, Gamper U, Boesiger P, Lomax A and Cattin P 2007 4D MR imaging of respiratory organ motion and its variability Physics in medicine and biology 521547

Welsh J, Gomez D, Palmer M B, Riley B A, Mayankkumar A V, Komaki R, Dong L, Zhu X R, Likhacheva A, Liao Z, Hofstetter W L, Ajani J A and Cox J D 2011 Intensity-Modulated Proton Therapy Further Reduces Normal Tissue Exposure During Definitive Therapy for Locally Advanced Distal Esophageal Tumors: A Dosimetric Study International Journal of Radiation Oncology*Biology*Physics 81 1336-42

Worm E S, Høyer M, Hansen R, Larsen L P, Weber B, Grau C and Poulsen P R 2018 A Prospective Cohort Study of Gated Stereotactic Liver Radiation Therapy Using Continuous Internal Electromagnetic Motion Monitoring International Journal of Radiation Oncology*Biology*Physics 101 366-75

Zhang X, Li Y, Pan X, Xiaoqiang L, Mohan R, Komaki R, Cox J D and Chang J Y 2018 Intensity-Modulated Proton Therapy Reduces the Dose to Normal Tissue Compared With Intensity-Modulated Radiation Therapy or Passive Scattering Proton Therapy and Enables Individualized Radical Radiotherapy for Extensive Stage IIIB Non-Small-Cell Lung Canc International Journal of Radiation Oncology • Biology • Physics 77 357-66

Zhang Y, Boye D, Tanner C, Lomax A J and Knopf A 2012 Respiratory liver motion estimation and its effect on scanned proton beam therapy Physics in medicine and biology 571779 